\title{
Application of Fuzzy Automata Decision-Making System in Target Control
}

\author{
Qinggang Wu${ }^{1}$, Zhenyu Han'2, Qing E. Wu ${ }^{3 *}$ \\ ${ }^{1}$ School of Computer and Communication Engineering, Zhengzhou University of Light Industry, Zhengzhou, China \\ ${ }^{2}$ Engineering Training Center, Zhengzhou University of Light Industry, Zhengzhou, China \\ ${ }^{3}$ College of Electric and Information Engineering, Zhengzhou University of Light Industry, Zhengzhou, China \\ Email:wuqinggang323@126.com,hzy196611@163.com, *wqe969699@163.com
}

How to cite this paper: Wu, Q.G., Han, Z.Y. and Wu, Q.E. (2017) Application of Fuzzy Automata Decision-Making System in Target Control. Journal of Computer and Communications, 5, 16-25. https://doi.org/10.4236/jcc.2017.510003

Received: July 24, 2017

Accepted: August 7, 2017

Published: August 10, 2017

Copyright $(9) 2017$ by authors and Scientific Research Publishing Inc. This work is licensed under the Creative Commons Attribution International License (CC BY 4.0).

http://creativecommons.org/licenses/by/4.0/

(c) (i) Open Access

\begin{abstract}
In order to perform better in target control, this paper proposed a decisionmaking system method based on fuzzy automata. The decision-making system first preprocessed the signal and then performed a two-level decision on the target to achieve optimal control. The system consisted of four parts: signal preprocessing, contrast decision-making, comprehensive judgment of decision-making and decision-making result. These decision algorithms in target control were given. A concrete application of this decision-making system in target control was described. Being compared with other existing methods, this paper used both global features and local features of target, and used the decision-making system of fuzzy automata for the target control. Simulation results showed that the control effect based on the decision-making system was better than that of the other existing methods. Not only it was faster, but also its correct control rate was higher to be $95.18 \%$ for the target control. This research on the control system not only developed the FA theory, but also strengthened its application scope in the field of control engineering.
\end{abstract}

\section{Keywords}

Decision-Making System, Comprehensive Judgment, Fuzzy Automata, Target Control

\section{Introduction}

At present, there are few researches on fuzzy signal processing based on inference system of fuzzy automata (FA) and fuzzy image comprehension in practical engineering application field. The intelligence of artificial intelligence system is mainly reflected in the ability to solve the problem of uncertainty, therefore, fuzzy reasoning, decision-making and evaluation is a core research of ar- 
tificial intelligence and expert system. In order to speed up to solve this problem, this paper studied the target control method based on fuzzy automata, because FA can more objectively process various ambiguous cases and complicated things. This study will provide a powerful tool for the control of complex systems, which have important theoretical and practical value.

Since some features of many objective things had the uncertainties and ambiguities, fuzzy sets (FS) and rough sets (RS) had been proposed previously. The separate FS and RS methods had been successfully applied in many fields such as pattern recognition, machine learning, decision support, process control, predictive modeling, and so on. Some relevant theories and some applications of FS had been discussed in the literature [1]. FS set up a bridge between the ambiguous signal and the real world described by classical mathematics, and there were a lot of new results which were presented usually in its margins and intersections. Those methods and the theories were maturing, and a variety of fuzzy mathematical theories [2] [3] [4] [5] had been presented. Especially, fuzzy control was used in a wide range of applications [6] [7]. In 1974, Mamdani who was a British scholar [8] first used the fuzzy controller composed of fuzzy control statement to steam turbine and boiler operation control, which was successful in the laboratory. From then on, the development of fuzzy mathematics is taking a road from the theory to the technology and then to the product.

With the development of information technology, more and more scholars had studied FA, and had achieved fruitful results in theories [9] [10] and applications [11] [12]. Giles et al. (1992) [13] used a complete gradient algorithm to derive a Tomita language. Since then, all network models referencing the secondorder feedback neural network had implied the network structure described by Giles. The experimental results showed that a second-order feedback neural network using a real-time, forward training algorithm could derive the formal grammar from the positive and negative string training samples by learning. They used a heuristic method during and after neural network which was trained to extract an automaton. However, when the input string was getting longer and longer, the stability problem of the automaton had arisen.

Due to the need of confidentiality of communication, military and so on, and the environment for each signal was increasingly complex so that the characteristic information of the target had some ambiguity. However, fuzzy automata [2]-[7] is a powerful tool to deal with fuzzy feature information. Based on this basis, this paper focuses on the establishment of target control system of fuzzy automata (FA). The system will be compared with the old method in the simulation. The simulation results show that its correct control rate is as high as $95.18 \%$.

\section{FA Target Control Method}

This paper presents a target control system based on fuzzy automata (FA). The system will carry better out the target control through the implementation of two- 
level decision-making method. In the two-level decision-making, the specific decision-making algorithm will be given, and it will be compared with the existing control methods in the simulation. Figure 1 is the FA control model. The system consists of four parts: signal preprocessing, contrast decision-making, comprehensive judgment decision-making and decision-making result.

\subsection{FA Binary Comparison Decision}

Assume the universal set $X=\left(x_{1}, x_{2}, \cdots, x_{n}\right)$ is $n$ selected programs. A fuzzy priority relation will be established in $n$ selected programs, that is, the comparison is first performed between each two, and then this comparison is fuzzed again. Further, the overall sequencing is given by using the fuzzy membership method, which is fuzzy binary contrast decision.

In comparison with $x_{i}$ and $x_{j}, r_{i j}$ is used to denote the degree of priority that $x_{i}$ compares with $x_{j}$, and $r_{i j}$ is requested to satisfy the following conditions:

1) $r_{i i}=1$ (Easy to calculate);

2) $0 \leq r_{i j} \leq 1$;

3) when $i \neq j, \quad r_{i j}+r_{j i}=1$.

The matrix $R=\left(r_{i j}\right)_{n \times n}$ that consists of $r_{i j}$ is called the fuzzy priority matrix, and the relation determined by the matrix is called the fuzzy priority relation.

The methods and steps of binary contrast decision-making are:

1) Establishment of fuzzy priority relation

The comparison is first performed between each two, then the fuzzy priority matrix is established as:

$$
R=\left(r_{i j}\right)_{n \times n}
$$

\section{2) Sequencing method}

Membership function method is given, that is, the fuzzy priority matrix is directly processed by appropriate mathematical method. Thus, the membership function of fuzzy priority set $A$ on $X$ is obtained, and then a certain order of the whole object can be carried out based on the degree of membership of each element and on merits or demerits. The usual approach is to take a small rule:

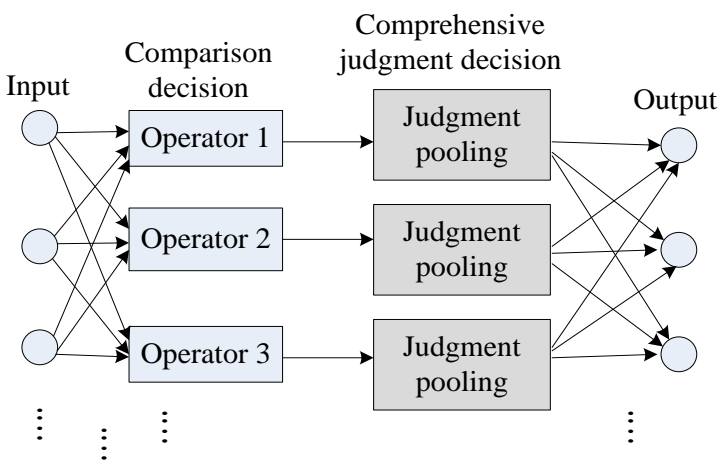

Figure 1. FA decision-making control system. 


$$
A\left(x_{i}\right)=\wedge\left\{r_{i j} \mid 1 \leq j \leq n\right\}, i=1,2, \cdots, n
$$

\subsection{FA Comprehensive Judgment Decision-Making}

For the evaluation or assessment of a thing, in reality, it often involves multiple factors or multiple indexes, then it is required to make a comprehensive evaluation for things based on various factors, and not to evaluate things only from a certain factor, which is a comprehensive judgment.

Fuzzy comprehensive judgment decision-making is a very effective multi-factor decision-making method for making a comprehensive evaluation to things affected by many factors.

Assume $U=\left(u_{1}, u_{2}, \cdots, u_{n}\right)$ is $n$ factors (or indexes), and $V=\left(v_{1}, v_{2}, \cdots, v_{m}\right)$ is $m$ kinds of judgment (or grades).

Because of different status of various factors, their roles are not the same, then the weight $A=\left(a_{1}, a_{2}, \cdots, a_{n}\right)$ can be used to describe these cases. The weight is a fuzzy subset in factor set $U$. For each factor $u_{i}$, a separate judgment $f\left(u_{i}\right)$ can be obtained, and seen as a fuzzy mapping $f$ from $U$ to $V$. A fuzzy relation $R_{f}$ can be induced by $f$ from $U$ to $V$. So, a fuzzy linear transformation can be induced by $R_{f}$ from $U$ to $V$ as follows:

$$
T_{R}(A)=A \circ R=B
$$

It is a fuzzy subset of the judgment set $V$, that is, a comprehensive judgment. Where $R=R_{f}$; 。 is a synthesis operation, which is the synthesis of the operation $\vee(\wedge)$.

$(U, V, R)$ forms the fuzzy comprehensive judgment decision model. $U, V$ and $R$ are the three elements of this model, respectively.

The method and procedure of fuzzy comprehensive judgment decision are:

1) To establish the factor set $U=\left(u_{1}, u_{2}, \cdots, u_{n}\right)$ and decision set $V=\left(v_{1}, v_{2}, \cdots, v_{m}\right)$.

2) To establish a fuzzy comprehensive judgment matrix.

For each factor $u_{i}$, a single factor judgment is first established as follows:

$$
\left(r_{i 1}, r_{i 2}, \cdots, r_{i m}\right)
$$

That is, $r_{i j}\left(0 \leq r_{i j} \leq 1\right)$ denotes the judgment of $v_{j}$ to the factor $u_{i}$, so that the single factor judgment matrix $R=\left(r_{i j}\right)_{n \times m}$ is obtained.

3) Comprehensive judgment.

A comprehensive judgment $B=A \oplus R=\left(b_{1}, b_{2}, \cdots, b_{m}\right)$ is carried out according to the weight $A=\left(a_{1}, a_{2}, \cdots, a_{n}\right)$ of each factor, which it is a fuzzy subset on $V$. The operation $\oplus$ can be defined based on the requirements of the actual problem. According to the different definitions of the operations $\oplus$, different decision models can be obtained. Here the operation $\oplus$ is defined as $\vee(\wedge)$, then the corresponding decision model is given as follows:

$$
b_{j}=\vee\left\{\left(a_{i} \wedge r_{i j}\right), 1 \leq i \leq n\right\}, j=1,2, \cdots, m
$$

Since the value of the result $b_{j}$ of comprehensive judgment is determined 
only by one of $a_{i}$ and $r_{i j}(i=1,2, \cdots, n)$, which takes first small and then the larger. The focus is on the main factor, and other factors have little effect on the result. This kind of operation sometimes makes the decision result difficult to distinguish. In this case, use the following model:

$$
b_{j}=\sum\left(a_{i} \wedge r_{i j}\right)
$$

In practice, if the main factor plays a leading role in the comprehensive judgment, it is recommended to adopt (3) or (4). However, when the model (3) fails, the model (4) is used.

\subsection{Determination of Weight}

In the fuzzy comprehensive judgment decision-making, the weight is essential, since it reflects the status or role of possession of each factor in the comprehensive decision-making process, it directly affects the results of comprehensive decision-making.

The weight given by the experience can reflect the actual situation to a certain extent, and the results of the judgments are also realistic, but it is often subjective, so it cannot objectively reflect the actual situation, thus, the results of the judgments may be "distorted". Here, a method to determine the weight is given as follows:

In the comprehensive judgment decision, if the comprehensive decision $B=\left(b_{1}, b_{2}, \cdots, b_{m}\right)$ is given, and the judgment matrix is $R=\left(r_{i j}\right)_{n \times m}$, then the weight distribution $X$ of each factor is

$$
X=\left(x_{1}, x_{2}, \cdots, x_{n}\right), \quad x_{k}=\wedge_{j=1}^{m}\left\{b_{j} \mid r_{k j}>b_{j}\right\}
$$

Here an approximate processing approach about the weight is given: assume that there is a set of alternative weight distribution plan $J=\left\{A_{1}, A_{2}, \cdots, A_{s}\right\}$. We choose a best weight distribution $A_{k}$ in $J$ so that the comprehensive judgment decision $B_{k}=A_{k} \circ R$ decided by $A_{k}$ is most close to $B$.

\section{FA Decision Control Example and Simulation}

\subsection{Control Example}

In here, the control to the target is achieved by target tracking.

Here, the control to targets is discussed with three types of modules of FA decision system to track four targets as an example, as shown in the Table 1 . The

Table 1. Control decisions of some type modules of FA decision system.

\begin{tabular}{cccccc}
\hline \multirow{2}{*}{$\begin{array}{c}\text { Universal Set } \\
\text { Target Types }\end{array}$} & Position & Speed & Acceleration & Angular Velocity & $\begin{array}{c}\text { Decision } \\
\text { Control Degree }\end{array}$ \\
\cline { 2 - 5 } 1 & 0.1 & 0.2 & 0.3 & 0.4 & not so good \\
2 & 0.1 & 0.5 & 0.2 & 0.2 & very good \\
3 & 0.4 & 0.35 & 0.15 & 0.1 & better \\
4 & 0.3 & 0.4 & 0.1 & 0.2 & very good \\
\hline
\end{tabular}


universal set is the type $1,2,3,4$ of targets, i.e., $\Omega=\{1,2,3,4\}$. The factor set $C=$ \{position, speed, acceleration, angular velocity\}, the control decision set $D=\{$ very good, better, not so good, not good \}, then, what is the decision rule?

Solution: To the target 1 , let a number of fine modules of FA decision system carry on the single factor control. At some moment, consider the position only, there is $20 \%$ modules of FA decision system that are very good to control targets, $50 \%$ modules of FA decision system control targets better, $20 \%$ modules of FA decision system do not control targets well and 10\% modules of FA decision system control targets poorly. Therefore, we can conclude: The position is $\mapsto(0.2,0.5,0.2,0.1)$.

Similarly, assume the speed is $\mapsto(0.7,0.2,0.1,0)$, the acceleration is $\mapsto(0,0.4,0.5,0.1)$, and the angular velocity is $\mapsto(0.2,0.3,0.5,0)$.

Accordingly, the control matrix is $R=\left(\begin{array}{cccc}0.2 & 0.5 & 0.2 & 0.1 \\ 0.7 & 0.2 & 0.1 & 0 \\ 0 & 0.4 & 0.5 & 0.1 \\ 0.2 & 0.3 & 0.5 & 0\end{array}\right)$.

Because of the different type modules of FA decision system, the weights given to the three factors of targets are also different. Let some type modules of FA decision system provide target 1 weight for $a_{1}=(0.1,0.2,0.3,0.4)$. According to these type modules of FA decision system controlling the target, the comprehensive control can be obtained $b_{1}=a_{1} \circ R=(0.2,0.3,0.4,0.1)$, where the synthetic operation $\circ$ is performed according to Formula (3). The control $b_{1}$ shows that the degree of "very good" is $20 \%$, "better" is $30 \%$, "not so good" is $40 \%$, and "not good" is $10 \%$. According to the maximum membership principle, the conclusion obtained is "not so good".

Likewise, to other three targets, assume the control matrix is also $R$. The weights that these type modules of FA decision system provide for the three targets are respectively:

$$
a_{2}=(0.1,0.5,0.2,0.2), a_{3}=(0.4,0.35,0.15,0.1), a_{4}=(0.3,0.4,0.1,0.2)
$$

Then, the corresponding synthesis controls are, respectively:

$$
\begin{aligned}
& b_{2}=a_{2} \circ R=(0.5,0.2,0.2,0.1) \\
& b_{3}=a_{3} \circ R=(0.35,0.4,0.2,0.1) \\
& b_{4}=a_{4} \circ R=(0.4,0.3,0.2,0.1)
\end{aligned}
$$

The obtained conclusion is "very good", "better", and "very good", respectively, as shown in the Table 1.

By genetic algorithm, the obtained total control is:

$$
b=b_{2}+b_{3}+b_{4}
$$

In the following, we verify whether or not the control matrix $R$ is the optimum control matrix to the target 2, 3 and 4, respectively. Whether the control matrix $R$ to control the "position" attribute of the target 2 is an optimal control or not is only given in here. 
To the target 2, through low-level, middle-level and high-level modules of FA decision system tracking it, its position-evaluated vector can be obtained as follows, respectively:

$$
\begin{aligned}
& \beta_{1}=(0.2,0.3,0.3,0.2) \\
& \beta_{2}=(0.4,0.3,0.2,0.1) \\
& \beta_{3}=(0.7,0.1,0.1,0.1)
\end{aligned}
$$

Let the position vector corresponding to the matrix $R$ be $\beta=(0.6,0.2,0.1,0.1)$. According to the close processing method to the Formula (5), the degrees that $\beta$ is close to $\beta_{1}, \beta_{2}$ and $\beta_{3}$ are calculated as, respectively:

$$
\begin{aligned}
N\left(\beta_{1}, \beta\right) & =1-\frac{1}{4} \sum_{i=1}^{4}\left|\beta_{1}\left(u_{1}\right)-\beta\left(u_{1}\right)\right| \\
& =1-\frac{1}{4}(|0.2-0.6|+|0.3-0.2|+|0.3-0.1|+|0.2-0.1|)=0.80, u_{1} \in \Omega .
\end{aligned}
$$

Similarly, there are $N\left(\beta_{2}, \beta\right)=0.90, N\left(\beta_{3}, \beta\right)=0.95$.

According to the selected-near principle, the vector $\beta$ is very close to $\beta_{3}$, and then $\beta$ is controlled by the high-level modules of FA decision system, so it is an optimal control vector.

From the above calculation of $b_{1}, b_{2}, b_{3}$ and $b_{4}$ obtained, the equivalence class of $C$ and $D$ is obtained as follows, respectively:

$U / C=\left\{X_{1}, X_{2}, X_{3}, X_{4}\right\}$, where $X_{1}=\{1\}, X_{2}=\{2\}, X_{3}=\{3\}, X_{4}=\{4\}$

$U / D=\left\{Y_{1}, Y_{2}, Y_{3}\right\}$, where $Y_{1}=\{1\}, Y_{2}=\{3\}, Y_{3}=\{2,4\}$.

Then the decision rule is:

$r_{11}$ : IF the degree of membership of the position is minimal, THEN the control of modules of FA decision system to the targets is not so good, and the decision degree is: $\rho\left(X_{1}, Y_{1}\right)=\left|Y_{1} \cap X_{1}\right| /\left|X_{1}\right|=1$.

Similarly, there are the rules $r_{23}, r_{32}$ and $r_{43}$, and the corresponding decision degree is $\mu\left(X_{2}, Y_{3}\right)=1, \mu\left(X_{3}, Y_{2}\right)=1$ and $\mu\left(X_{4}, Y_{3}\right)=1$, respectively.

The general rules of modules of FA decision system to the target control are:

Let the control of modules of FA decision system to targets be "better" act as a criterion whether the modules of FA decision system are selected or not. According to the genetic algorithm, the "better" and "very good" modules of FA decision system are carried on the genetic algorithm combination. Thus, the fine modules of FA decision system association are obtained so as to accomplish better the control of targets.

According to the above rules, the rules $r_{23}, r_{32}$ and $r_{43}$ can be selected. According to the above Equation (6) again, if the state of motion of the target is a vector $X$, then the state of the target is controlled by $X_{t}=b \circ X$ based on the above control rules at the time $t$.

\subsection{Simulation}

For simplifying in simulation, a case that three modules of FA decision system to control an individual target is given in here. The actual weight of the target is 
$a=(0.2,0.3,0.3,0.2)$. Let the target make rectilinear motion at a uniform velocity and take a turn to the right or the left motion at a uniform velocity, where the deviation turning rates are $\omega_{1}=5^{\circ}$ or $\omega_{1}=-5^{\circ}$. The control weights of the modules 1,2 and 3 of FA decision system to the target are $a_{1}=(0.4,0.3,0.2,0.1)$, $a_{2}=(0.1,0.3,0.5,0.1)$ and $a_{3}=(0.1,0.4,0.3,0.2)$, respectively. The control matrix of systems is:

$$
\Psi=\left[\begin{array}{ccc}
0.3^{-\omega_{1} t} & 0.3^{-\omega_{1}(t+1)} & \ldots \\
0.3^{\omega_{2} t} & 0.3^{\omega_{2}(t+1)} & \ldots \\
0.3^{-\omega_{3} t} & 0.3^{-\omega_{3}(t+1)} & \ldots \\
1-0.3^{\omega_{2} t} & 1-0.3^{\omega_{2}(t+1)} & \ldots
\end{array}\right]
$$

where $\omega_{1}=5, \omega_{2}=-5 . t$ is a time variable. $\Psi$ is a $4 \times T_{0}$ matrix. The simulation sampling $T_{0}$ is 150 times, and the sampling rate $T$ is 2 seconds. The simulation results are shown in Figure 2.

From Figure 2(a), the FA decision-making system method proposed in this paper is better than the traditional methods [8] [12] for the tracking control to the target, and the tracking curve of the FA decision system method is almost close to the true orbit of the target. From Figure 2(b), to error tracking curve of the difference of estimate value and true value, the tracking error of FA decision system reduces gradually and trends towards stability. The mean-square error curve of FA decision system is shown in Figure 2(b).

Through the obtained data results in experiment process, the average accuracy rate and processing speed have been obtained based on 150 times simulations. To compare with the traditional control methods [8] [12], FA decision system

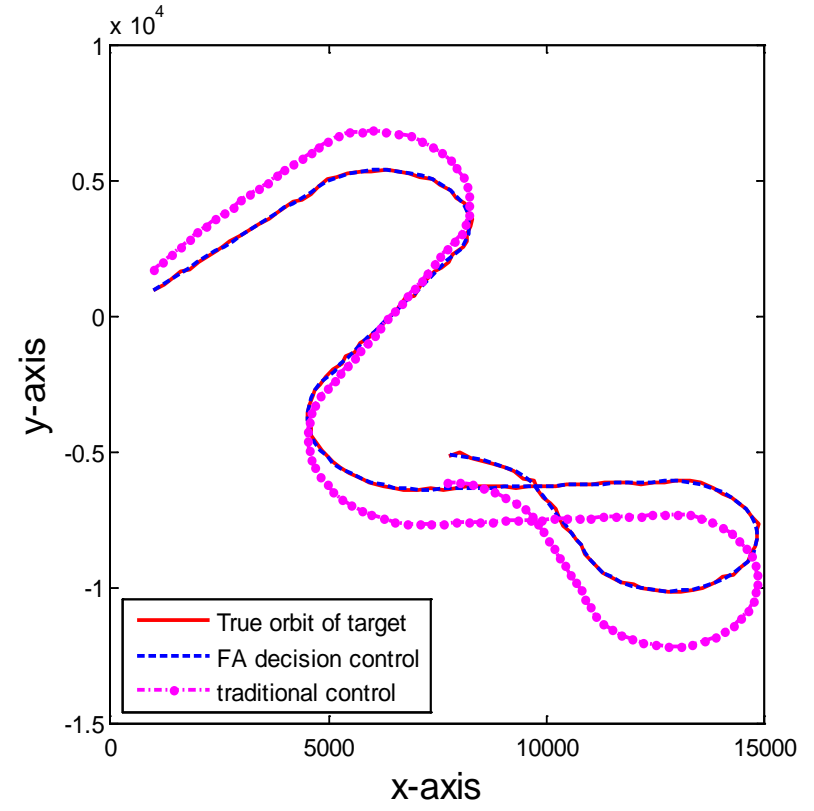

(a)

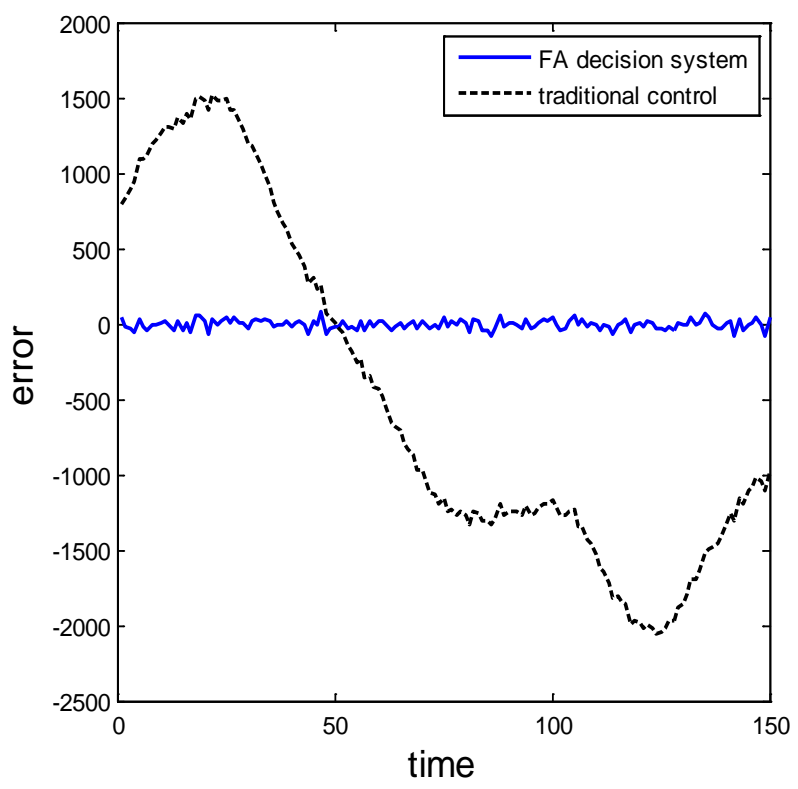

(b)

Figure 2. Comparison of FA decision system and traditional control methods. (a) Tracking control of FA decision and existing method; (b) Control error of FA decision and existing method. 


\section{Control precision of proposed and traditional control methods}

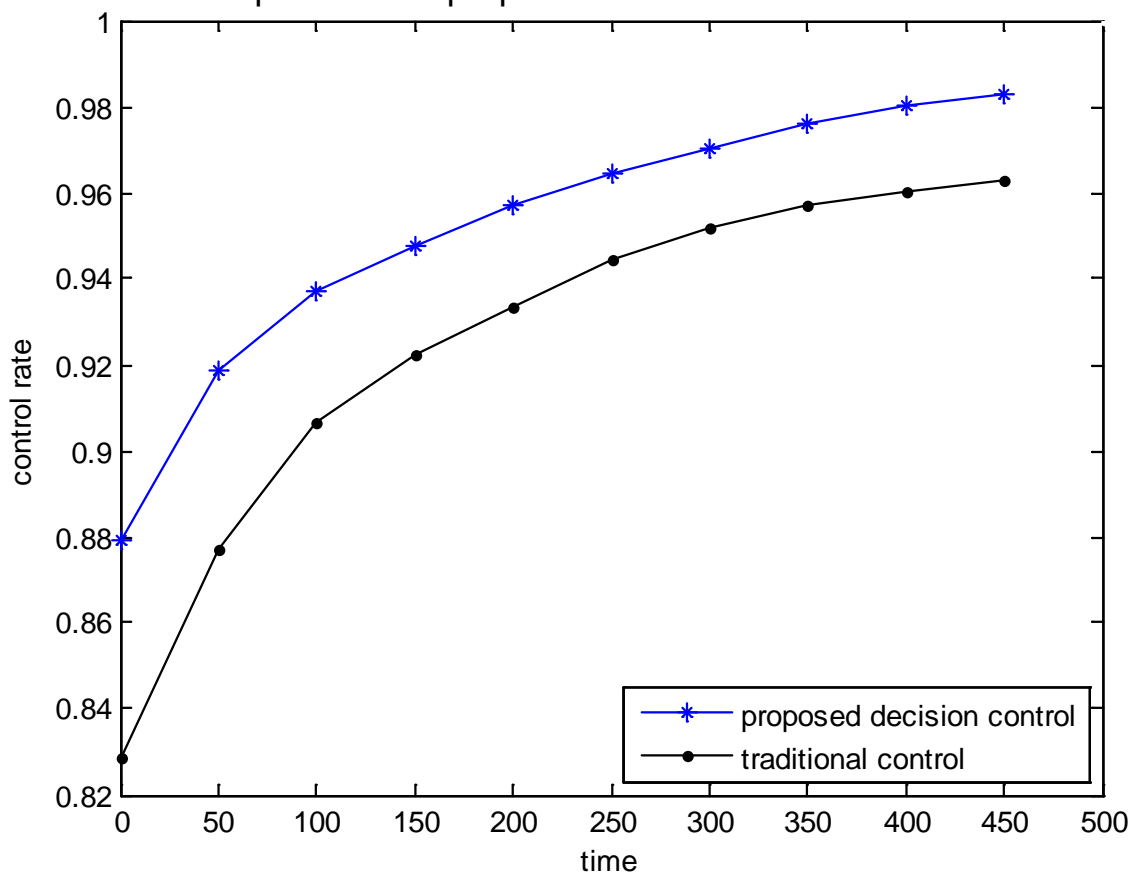

Figure 3. Comparison of accuracy of FA decision system and traditional control methods for target control.

method not only has faster processing speed, lower information exchange rate, but also has better control effect and its control precision is averagely $95.18 \%$, however, that of the traditional method is $90.96 \%$ on average. The average accuracy rate of the proposed method is higher by $4.22 \%$ than that of the traditional control method. The part results in simulation are shown in Figure 3. At the same time, its control speed is faster by $9.8 \mathrm{~s}$ than that of the traditional control method.

\section{Conclusion}

Based on the theory of FA, this paper proposes the control method of FA decision system to target control, gives the FA decision system model, and gives the two-level decision algorithms. At the same time, the decision rules and decision degree of FA decision-making system are discussed. Finally, the application of FA decision-making system in target control is described by examples. The simulation results show that it is faster and has better control effect for target tracking control, compared with the traditional control methods, because the control precision of the proposed FA decision system is averagely $95.18 \%$, which is higher by $4.22 \%$ than that of the traditional control method. At the same time, its control speed is faster by $9.8 \mathrm{~s}$ than that of the traditional control method.

\section{Acknowledgements}

This work is supported by Henan Province Outstanding Youth on Science and Technology Innovation (No. 164100510017); National 973 Program (No. 613237); 
National Natural Science Foundation of China (No. 61502435); Key Science and Technology Program of Henan Province Education Department (No. 14A520034);

Doctorate Research Funding and Project of Key Young Teachers of ZZULI (No. 2013BSJJ041, 13300093), respectively.

\section{References}

[1] Yang, L. and Gao, Y. (2002) Fuzzy Mathematics Principle and Application. South China University of Technology Press, Guangzhou. (In Chinese)

[2] Shamsizadeh, M. (2016) Intuitionistic General Fuzzy Automata. Soft Computing, 20, 291-293. https://doi.org/10.1007/s00500-015-1969-x

[3] Karthikeyan, V. (2015) Directable Fuzzy Automata. International Journal of Computer Applications, 125, 1-4. https://doi.org/10.5120/ijca2015906119

[4] Li, Y.M. and Wang, Q. (2014) The Universal Fuzzy Automaton. Fuzzy Sets and Systems, 249, 27-48. https://doi.org/10.1016/j.fss.2013.08.002

[5] Pan, H., Li, Y., Cao, Y. and Li, P. (2017) Nondeterministic Fuzzy Automata with Membership Values in Complete Residuated Lattices. International Journal of Approximate Reasoning, 82, 22-38. https://doi.org/10.1016/j.ijar.2016.11.020

[6] Micic, I., Jancic, Z., Ignjatovic, J. and Ciric, M. (2015) Determinization of Fuzzy Automata by Means of the Degrees of Language Inclusion. IEEE Transactions on Fuzzy Systems, 23, 2144-2153. https://doi.org/10.1109/TFUZZ.2015.2404348

[7] Garhwal, S. and Jiwari, R. (2016) Conversion of Fuzzy Automata into Fuzzy Regular Expressions Using Transitive Closure. Journal of Intelligent and Fuzzy Systems, 30, 3123-3129. https://doi.org/10.3233/IFS-152038

[8] Mamdani, E.H. (1974) Applications of Fuzzy Algorithms for Simple Dynamic Plant. IEEE on Control, 121, 1585-1588. https://doi.org/10.1049/piee.1974.0328

[9] Jancic, Z., Micic, I., Ignjatovic, J. and Ciric, M. (2016) Further Improvements of Determinization Methods for Fuzzy Finite Automata. Fuzzy Sets and Systems, 301, 79-102. https://doi.org/10.1016/j.fss.2015.11.019

[10] Li, L. and Qiu, D. (2015) On the State Minimization of Fuzzy Automata. IEEE Transactions on Fuzzy Systems, 23, 434-443. https://doi.org/10.1109/TFUZZ.2014.2315620

[11] Zeng, Z., Goodman, R. and Smyth, P. (1993) Learning Finite State Machines with Self-Clustering Recurrent Networks. Neural Computation, 5, 976-990. https://doi.org/10.1162/neco.1993.5.6.976

[12] Garitagoitia, J.R., Mendívil, J.R.G.D., Echanobe, J., et al. (2003) Deformed Fuzzy Automata for Correcting Imperfect Strings of Fuzzy Symbols. IEEE Transactions on Fuzzy Systems, 11, 299-310. https://doi.org/10.1109/TFUZZ.2003.812682

[13] Giles, C.L., Miller, C.B. and Chen, D. (1992) Learning and Extracting Finite State Automata with Second-Order Recurrent Neural Networks. Neural Computation, 4, 393-405. https://doi.org/10.1162/neco.1992.4.3.393 
Submit or recommend next manuscript to SCIRP and we will provide best service for you:

Accepting pre-submission inquiries through Email, Facebook, LinkedIn, Twitter, etc. A wide selection of journals (inclusive of 9 subjects, more than 200 journals)

Providing 24-hour high-quality service

User-friendly online submission system

Fair and swift peer-review system

Efficient typesetting and proofreading procedure

Display of the result of downloads and visits, as well as the number of cited articles Maximum dissemination of your research work

Submit your manuscript at: http://papersubmission.scirp.org/

Or contact jec@scirp.org 